\title{
From problem-solving to research utilization: How operations research and program evaluation can make programs better
}

Karen Foreit

Follow this and additional works at: https://knowledgecommons.popcouncil.org/departments_sbsr-hiv

Part of the Demography, Population, and Ecology Commons, Family, Life Course, and Society Commons, Health Policy Commons, International Public Health Commons, and the Medicine and Health Commons How does access to this work benefit you? Let us know!

\section{Recommended Citation}

Foreit, Karen. 2013. "From problem-solving to research utilization: How operations research and program evaluation can make programs better." Washington, DC: USAID I Project Search: HIVCore. 


\section{FROM PROBLEM-SOLVING TO RESEARCH UTILIZATION HOW OPERATIONS RESEARCH AND PROGRAM EVALUATION CAN MAKE PROGRAMS BETTER}

There is little doubt that HIV treatment, care and support, and prevention of mother-to-child transmission (PMTCT) programs are most effective when they are based on the best available research evidence.' This is the fundamental premise of evidence-based medicine. Equally well-known is the dilemma of transforming evidence into practice, which is the subject of knowledge translation and implementation science. Even when the findings of clinical trials make their way into international and national program guidelines, they run up against the reality of competing priorities within public health and community programs, resource constraints, and institutional and human inertia.

Understanding how to effectively change the approach used in program operations requires understanding how programs work in the first place. Policy makers, program managers, and service providers routinely confront two fundamental and inter-related questions:

I. Is the program working?

2. Could the program work better?

Answering these questions requires information, as does making a decision about the way forward -continue the program as is, change course, add a new component, etc. Most decisions are based on a combination of factors including personal experience, common sense, political realities, and program and/or research data. Operations research (OR) and program evaluation (PE) approaches can help managers be more systematic in examining existing program information, collecting new data if needed and looking for alternative solutions.

\section{STANDARDS OF EVIDENCE, RESEARCH DESIGN,AND ETHICS}

There are costs involved both in making decisions without appropriate information and in systematically collecting and analyzing data for informed decisionmaking. The challenge for program managers, researchers, and evaluators is to produce data that are good enough to answer the question at hand in a cost-effective manner that respects the privacy and confidentiality of program beneficiaries.

What is good enough? Data requirements-precision and generalizability-should be guided by the context of the question and the cost of correcting a mistake.

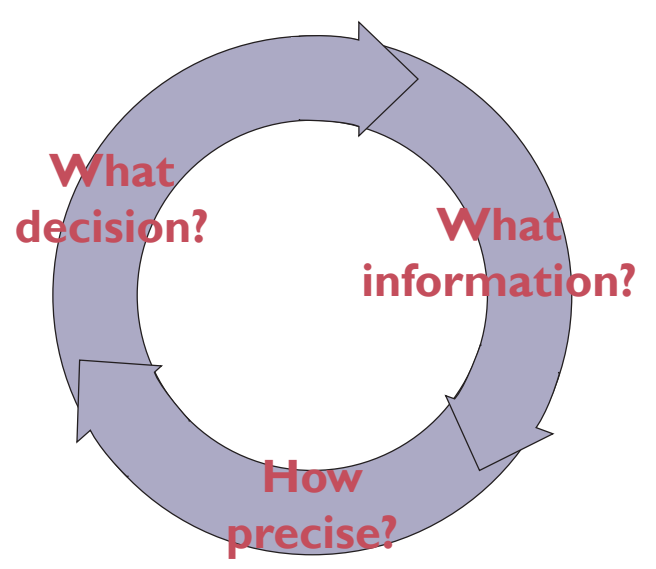


A quick review of a handful of records or observation of a clinic waiting room may be enough to spot operational bottlenecks, and service providers may be able to come up with possible solutions on the spot and try them for a short period of time to see if they work. On the other hand, before scaling up a promising pilot project, policy makers may want more substantial evidence that the new procedures not only produce better outcomes but that they are less costly than current program norms. Convincing policy makers to undertake costly and time-consuming program changes and overcome entrenched interests often requires a well-designed experimental study with comparison or control groups to demonstrate the counter-factual, along with political advocacy to promote utilization of the research findings.

"You can't shift policies overnight, when dealing with a large network of health facilities. When you want to change policy you have to think two or three years in advance. You have to train people, run seminars, change curricula, explain why you want to change, in some cases mount a different logistic system. This may be very costly and therefore you should have good reasons to change."

To reduce costs of data collection, preference should be given to utilizing existing program data whenever possible. Depending on the setting, these may run the gamut from individual medical records and/or family folders to daily logs to monthly summary reports. Where these data are inaccessible, incomplete, or problematic for other reasons, program managers and researchers should look for quick and low-cost data collection methods, for example, client intercept surveys to supplement program registers.

The minimum design for testing a solution to a program problem is some kind of before- and after-intervention measurement. Oftentimes a simple time-series analysis will be sufficient, especially if the scope of the intervention is limited to a single site or clinic.

If sufficient pre-intervention baseline data are available, time-series analysis may be sufficient for a full-fledged OR study. For example, a vasectomy clinic had been
In Mozambique, HIV patients need a CD4 test before starting treatment. Staff noticed that delays in physicians' ordering the test were delaying initiation of treatment. Since all patients need to be tested, they decided to have the receptionist order the tests. Improvements in testing were seen so quickly that receptionist-ordered testing was adopted as a standard practice.

operating for several years in São Paulo, Brazil, and productivity had stabilized at a level below installed capacity. The clinic director obtained an OR project to support a short advertising campaign in local magazines. The project supplemented clinic service statistics with interviews asking new clients how they obtained the clinic's phone number and, if they mentioned a magazine ad, which magazine. Figure I presents the findings. While there was considerable month-tomonth variation in clinic performance, even without statistical analysis it is clearly evident that clinic performance increased following the advertising campaign; that the advertising was responsible for the increase was corroborated by the client interviews. ${ }^{3}$

\section{Figure I Mean daily procedures performed} pre- and post-intervention

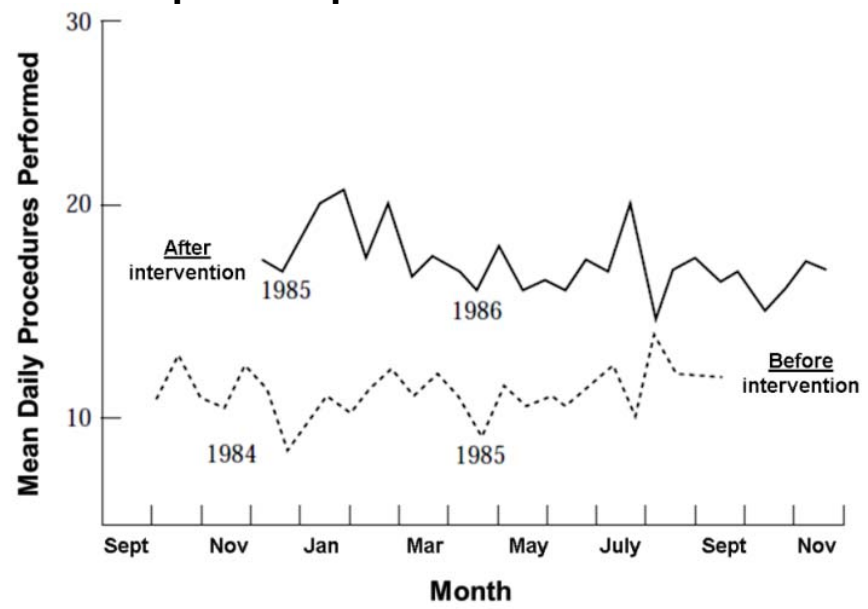

Regardless of the data sources and data collection paradigm, researchers and evaluators should always behave ethically. Whether formal approval from an Institutional Review Board (IRB) is needed depends on the regulatory climate of the country, the source of 
funding (if any), the intent of the inquiry, the nature of the data and data collection procedures, and the identity of those who will have access to the data. Research funded through HIVCore - as with any other research funded by the U.S. government-must follow U.S. federal regulations protecting human subjects. ${ }^{4}$ HIVCore protocols must be reviewed and approved both in the host country and in the U.S.

A common ethical issue in OR and PE is protecting private, identifiable information, as in the case of medical records. As a rule of thumb, program managers and service providers usually would not need IRB approval to review their own clinical records to identify and solve problems in their own facilities, provided that they do not reveal clients' identities in reports or dissemination of the findings. Larger-scale medical record review or new data collection would usually need IRB oversight and client interviews would need informed consent. Moreover, researchers should keep in mind that professional peer-reviewed journals routinely require evidence that research ethics procedures were followed, as can be seen in the requirements imposed by the Journal of Acquired Immune Deficiency Syndromes on authors submitting manuscripts for publication.

"When reporting experiments involving human subjects, authors should indicate whether the procedures followed were in accordance with the ethical standards of the responsible committee on human experimentation (institutional and national) and with the Helsinki Declaration of 1975, as revised in 2000." ${ }^{5}$

\section{HIVCORE'S MANDATE}

HIVCore encompasses PE and OR in the context of health system strengthening in general and HIV treatment, care and support, and PMTCT in particular. Program issues of interest may include quality, efficiency or cost-efficiency, and overall outcomes or impacts. It should also be noted that HIVCore's mandate does not include project-level evaluation. ${ }^{6}$

Our application of OR focuses on factors under the control of and which can be manipulated by program managers and on indicators of program success and/or cost-effectiveness of program operations. ${ }^{7}$ This focus does not argue that factors not under the control of managers are unimportant. On the contrary, the program manager's responsibility is to design and test interventions to overcome the barriers posed by these contextual factors. Contextual factors hindering access could include community factors such as lack of transport, socio-cultural factors such as women needing permission to seek services, or economic factors such as lack of money to pay for services. Thus, research that tests strategies for removing barriers to use, such as network expansion to provide services in rural areas, may qualify as OR.

HIVCore's application of OR not only links research to practice, but also practice to research. We begin by determining whether a particular problem can be solved through common sense or experience, through the application of lessons learned from past OR studies or reanalysis of existing data. Only after these alternatives have been explored will we design studies involving new data collection.

While OR tends to be forward-looking, PE is usually retrospective. Evaluation asks the "so what" question: did the program accomplish what it intended to accomplish? HIVCore focuses on basic PE, which seeks to answer descriptive questions related to program design, management, and operational decision making such as: what has the program achieved; how is it being implemented; how is it perceived and valued; what are its unintended consequences, whether positive or negative; and whether expected performance benchmarks are being met.

HIVCore OR and PE will make use of both secondary analyses of existing data sets (e.g., clinical records, program registers) as well as primary data collection. To demonstrate the effect or value of program interventions, HIVCore will include indicators of service delivery, community support, and/or client outcomes. In many cases, client outcomes will be measured by broad programmatic outcomes such as getting a key service such as HIV or CD4 testing, initiating pre-treatment care, initiating treatment, and retention of treatment. Unless the data are readily available, HIVCore OR 
studies typically will not include clinical outcomes such as a fall in viral load, a rise in CD4 counts, or a gain in body weight.

Within the project's time and budget constraints, OR studies may include both formative and/or intervention research. The programmatic implications or applications of research findings should be specified in advance.

- What is the specific program problem?

- Example: failure to enroll people testing HIVpositive into treatment programs.

- What are the larger implications of this problem?

- Poorer client outcomes due to late enrollment into treatment.

- Inefficient use of program resources.

- How widespread or profound is the problem?

- Secondary analysis of existing data.

- Focused formative research.

- How will we know if the problem has been solved?

- Selection of dependent variable(s). In selecting dependent variables, especially in intervention studies, preference will be given to outcomes that can show a programmatically important, measurable change in a short period of time.

- Time will be needed to conduct in-country ancillary activities, including:

- Protocol development workshop with local researchers and program managers.

- Data analysis workshop.

- Data interpretation meeting for stakeholders.

- Writing workshop.

\section{UTILIZATION OF RESEARCH AND EVALUATION FINDINGS}

Testing a successful program intervention does not equal successful operations research. $O R$ is successful if and when program managers and policy makers consider the results from the research to continue or scale up interventions that improve program effectiveness or efficiency, or to abandon interventions that do not. Findings are more likely to be used if researchers, managers, and other stakeholders are involved in the research process from the very beginning. ${ }^{8}$

While this document has focused on research and evaluation considerations, HIVCore's mandate goes beyond implementing OR and PE. It also includes building local capacity to identify operational issues and conduct appropriate research to answer them, and widely disseminating the findings. In this way, we hope that HIVCore will help program managers and policy makers decide between alternative courses of action, identify and take advantage of opportunities, and find solutions to service-delivery problems that limit program effectiveness and efficiency.

\footnotetext{
'P. Glasziou and B. Haynes. 2005. "The paths from research to improved health outcomes," American College of Physicians Journal Club I42:A8-AI0.

${ }^{2}$ Former Minister of Health of Mozambique, cited in J. Cliff, G. Watt, and I. Nhatave. 2004."What's in a name? Policy transfer in Mozambique: DOTS for tuberculosis and syndromic management for sexually transmitted infections," Journal of Public Health Policy 25: 38-55.

${ }^{3}$ Foreit, K. G., M. P. P. de Castro and E. F. D. Franco. 1989. "The impact of mass media advertising on a voluntary sterilization program in Brazil," Studies in Family Planning 20: 107-1 16.

${ }^{4} 45$ CFR 46. http://www.hhs.gov/ohrp/humansubjects/guidance/45cfr $46 . \mathrm{html}$

5Journal of Acquired Immune Deficiency Syndromes. 20I3. "Online submission and review system." http://edmgr.ovid.com/jaids/accounts/ifauth.htm.

'USAID. 20II. "Evaluation learning from experience," USAID Evaluation Policy, p.8. http://transition.usaid.gov/evaluation/USAIDEvaluationPolicy.pdf

${ }^{7}$ Foreit, J. R. and T. Frejka (Eds.). 1998. Family Planning Operations Research:A Book of Readings. New York: Population Council.

${ }^{8}$ P. Davis and P. Howden-Chapman. 1996."Translating research findings into health policy," Social Science and Medicine 43: 865-872.
}

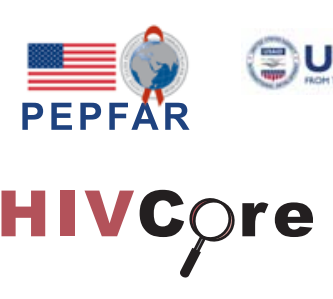

USAID |Project SEARCH

HIVCore improves the efficiency, effectiveness, scale, and quality of HIV treatment, care, and support, and prevention of mother-to-child transmission (PMTCT) programs. The Task Order is led by the Population Council in partnership with the Futures Group, the Elizabeth Glaser Pediatric AIDS Foundation, and the University of Washington. www.hivcore.org 